\title{
Debye mass and heavy quark potential in a PNJL quark plasma
}

\author{
J. Jankowski ${ }^{1, *}$ and D. Blaschke $e^{1,2,+\dagger}$ \\ ${ }^{1}$ Institute for Theoretical Physics, University of Wroclaw, 50-204 Wroclaw, Poland \\ ${ }^{2}$ Bogoliubov Laboratory of Theoretical Physics, JINR Dubna, 141980 Dubna, Russia \\ We calculate the Debye mass for the screening of the heavy quark potential in a \\ plasma of massless quarks coupled to the temporal gluon background governed by \\ the Polyakov loop potential within the PNJL model in RPA approximation. We give \\ a physical motivation for a recent phenomenological fit of lattice data by applying \\ the calculated Debye mass with its suppression in the confined phase due to the \\ Polyakov-loop to a description of the temperature dependence of the singlet free \\ energy for QCD with a heavy quark pair at infinite separation. We compare the \\ result to lattice data.
}

\section{INTRODUCTION}

The proposal of Matsui and Satz [1] that color screening of heavy quarks in a deconfined medium should lead to a dissociation of the $J / \psi$ bound state and whence could be a clear signal of quark-gluon plasma (QGP) formation in heavy-ion collision (HIC) experiments arose to a wide field of research both in experimental as well as theoretical directions [2]. Following the original idea the simplest picture is to consider a static quark-antiquark $(Q \bar{Q})$ probe in a color plasma where all medium effects are included in two body interaction potential $V_{T}(r)$. Then solving the $Q \bar{Q} T$-matrix [3] for such a system (or equivalently Schrödinger equation [4]) one can obtain a temperature modification of the hadron spectra. For obvious reasons this description is unrealistic (e.g., in HIC experiments the medium always has collective flow) but nevertheless it can provide physically reliable insights. But what potential should we use? One possibility of approaching this problem is to use nonperturbative field theoretical methods to motivate an ansatz for the heavy quark potential and then fit its

*Electronic address: jakubj@ift.uni.wroc.pl

${ }^{\dagger}$ Electronic address: blaschke@ift.uni.wroc.pl 
parameters to the lattice data. For example in ref. [3, 5] it has been shown that including effects of a dimension-two gluon condensate one gets quarkonium spectral functions in good agreement with lattice data.

In this note we go similar way by investigating Debye screening effects in modifying the vacuum Cornell potential (one-gluon exchange plus linear term) when going to a finite temperature medium. The screening medium is composed of massless quarks coupled to the homogeneous, temporal gluon background which is a very crude approximation of confinement. This essentially constitutes a version of the PNJL model [8, 9] which was found to reproduce lattice thermodynamics although it is theoretically still rather unsatisfactory. In this contribution we discuss the similarities of the temperature dependence of the Debye mass within such a model and that obtained from a fit of lattice QCD heavy quark free energy with the effective interaction of Ref. [3]. This observation holds promise for a better microscopic understanding of the physical effects determining the behaviour of the heavy quark-antiquark potential at finite temperature.

\section{SCREENING IN THE GLUON BACKGROUND}

In Ref. [6] a model was considered where the vacuum potential was screened by quark matter polarization decribed by quark-antiquark loop integrals where the internal lines were coupled to a temporal gluon background field. Specifically, when the static interaction potential is given as $V(q), q^{2}=|\mathbf{q}|^{2}$, the statically screened potential is given by a resummation of one-particle irreducible diagrams ("bubble" resummation $=\mathrm{RPA}$ )

$$
V_{\mathrm{sc}}(q)=V(q) /\left[1-\Pi_{00}(0 ; \mathbf{q}) / q^{2}\right],
$$

where the longitudinal polarization function in the finite $T$ case can be calculated within thermal field theory as

$$
\Pi_{00}\left(i \omega_{l} ; \mathbf{q}\right)=g^{2} T \sum_{n=-\infty}^{\infty} \int \frac{d^{3} p}{(2 \pi)^{3}} \operatorname{Tr}\left[\gamma^{0} S_{\Phi}\left(i \omega_{n} ; \mathbf{p}\right) \gamma^{0} S_{\Phi}\left(i \omega_{n}-i \omega_{l} ; \mathbf{p}-\mathbf{q}\right)\right] .
$$

Here $\omega_{l}=2 \pi l T$ are the bosonic and $\omega_{n}=(2 n+1) \pi T$ are the fermionic Matsubara frequencies of the imaginary-time formalism. The symbol Tr stands for traces in color, flavor and Dirac spaces. $S_{\Phi}$ is the propagator of a massless fermion coupled to the homogeneous static gluon background field $\varphi_{3}$. Its inverse is given by $[8,9]$

$$
S_{\Phi}^{-1}\left(\mathbf{p} ; \omega_{n}\right)=\gamma \cdot \mathbf{p}+\gamma_{0} i \omega_{n}-\gamma_{0} \lambda_{3} \varphi_{3},
$$


where $\varphi_{3}$ is related to the Polyakov loop variable defined by [8]

$$
\Phi(T)=\frac{1}{3} \operatorname{Tr}_{\mathrm{c}}\left(\mathrm{e}^{\mathrm{i} \beta \lambda_{3} \varphi_{3}}\right)=\frac{1}{3}\left(1+2 \cos \left(\beta \varphi_{3}\right)\right) .
$$

The physics of $\Phi(T)$ is governed by the temperature-dependent Polyakov loop potential $\mathcal{U}(\Phi)$, which is fitted to describe the lattice data for the pressure of the pure glue system [8]. After performing the color-, flavor- and Dirac traces and making the fermionic Matsubara summation, we obtain in the static, long wavelength limit

$$
\Pi_{00}(\mathbf{q})=\frac{2 N_{c} N_{f} g^{2}}{\pi^{2}} \int_{0}^{\infty} d p p^{2} \frac{\partial f_{\Phi}}{\partial p}=-2 g^{2} T^{2} I(\Phi)=-m_{D}^{2}(T),
$$

where $m_{D}(T)$ is the Debye mass, the number of degrees of freedom is $N_{c}=3, N_{f}=2$ and $f_{\Phi}(p)$ is the quark distribution function [9]. In comparison to the free fermion case [10, 11] the coupling to the Polyakov loop variable $\Phi(T)$ gives rise to a modification of the Debye mass, given by the integral

$$
I(\Phi)=\frac{12}{\pi^{2}} \int_{0}^{\infty} d x x \frac{\Phi\left(1+2 e^{-x}\right) e^{-x}+e^{-3 x}}{1+3 \Phi\left(1+e^{-x}\right) e^{-x}+e^{-3 x}} .
$$

The temperature dependence of $\Phi(T)$ is taken from Ref. [12]. In the limit of deconfinement $(\Phi=1)$, the case of a massless quark gas is obtained $(I(1)=1)$, while for confinement $(\Phi=0)$ one finds that $I(0)=1 / 9$. The temperature dependence of the resulting Debye mass is shown in Fig. 1 1 and as expected from the very beginning is much lower, comparing to the free, massless case, in the confined and transition region (with the critical temperature $T_{c} \approx$ $200 \mathrm{MeV}$ ). For temperatures $T>>T_{c}$ the free gas behavior is reproduced. Adopting the field theoretical approach of Ref. [3, 5] that the color singlet free energy at finite temperature is driven by the screened, nonperturbative gluon propagator and that Debye masses in Coulomb and stringy sectors are different, we get $\left(\alpha_{s}=g^{2} / 4 \pi\right)$

$$
F_{\overline{\mathrm{Q}} \mathrm{Q}}(r)=-\frac{4}{3} \alpha_{s}\left(\frac{e^{-m_{D} r}}{r}-\frac{m_{G}^{2}}{2 \widetilde{m}_{D}}+\frac{m_{G}^{2}}{2 \widetilde{m}_{D}} e^{-\widetilde{m}_{D} r}+m_{D}\right),
$$

where the constant ( $r$ - independent) term is a homogeneous mean field contribution and takes into account the one-particle self-energy effects [2, 4]. We take $m_{G}^{2}=0.631 \mathrm{GeV}^{2}$ which gives string tension $\sigma=2 m_{G}^{2} \alpha_{s} / 3=0.198 \mathrm{GeV}^{2}$. We notice that our calculated Debye mass resembles qualitatively the behavior resulting from a recent fit for the stringy term in Ref. [3] with the difference that our result is smaller than that of the fit by abut a factor two. Therefore we proceed by plugging it in for $\widetilde{m_{D}}$ in equation (6), and if we define

$$
F_{\infty}(T)=F_{\bar{Q} Q}(r \rightarrow \infty)=\frac{4}{3} \alpha_{s}\left(\frac{m_{G}^{2}}{2 \widetilde{m}_{D}}-m_{D}\right) .
$$


Then we see that $F_{\infty}(T \rightarrow \infty) \approx-T$, and that it diverges for small temperatures - faster than in the free fermion case due to gluon suppression of $\widetilde{m}_{D}$ - which is expected in quenched approximation but not in QCD with dynamical quarks which would make $F_{\infty}$ finite due to so called "string breaking mechanism". Fitting lattice data for $F_{\infty}(T)$ [7] for high temperatures results in a coulombic Debye mass $m_{D}=0.12 T$ which is much lower than the perturbative one which is about $3.143 T$ for two flavors and $\alpha_{s}=0.471$. We plot the resulting free energies in Fig. 1. What concerns $F_{\infty}(T)$ we see that our results meet quite well the lattice data in a quite a wide temperature range starting from the transition region up to high temperatures; the generic shape is reproduced in the transition region, while we see a discrepancy for low temperatures. Our result is smaller than the lattice (especially for low $T$ ) which is a remnant of our lower Debye mass than the one of the fit by Riek and Rapp [2]. What concerns the free energy for small distances, it is temperature independent and matches $T=0$ potential, while $r \approx 0.25 \mathrm{fm}$ marks the onset of temperature effects. The temperature dependence of binding energies obtained with this approach suggests [6] that charmonium (bottomonium) dissociation takes place at $T \approx 200 \mathrm{MeV}(T \approx 250 \mathrm{MeV})$.

\section{CONCLUSIONS}

It is clearly shown that even a crude inclusion of residual effects from the gluon sector results in a remarkable improvement. One of the obvious extensions of this calculation is to take into account chiral symmetry effects on the internal quark lines, governed in this model

by the local NJL interaction [9]. In this way one could in principle study the effects of the chiral transition on $J / \psi$ dissociation.

\section{Acknowledgments}

We acknowledge inspiring discussions with our colleagues, in particular K. Redlich, O. Kaczmarek and R. Rapp. J.J. is grateful for support of his participation at the CPOD 2010 conference from HIC-for-FAIR and the Bogoliubov-Infeld Programme. This work was supported in part by the Polish Ministry for Science and Higher Education and by the Russian Fund for Basic Research under grant No. 11-02-01538-a. 
[1] T. Matsui and H. Satz, Phys. Lett. B 178416 (1986).

[2] R. Rapp, D. Blaschke and P. Crochet, Prog. Part. Nucl. Phys. 65 (2010) 209 arXiv:0807.2470 [hep-ph]].

[3] F. Riek and R. Rapp, Phys. Rev. C 82 (2010) 035201 arXiv:1005.0769 [hep-ph]].

[4] W. Ebeling, W.-D. Kraeft, D. Kremp, G. Röpke, Quantum Statistics of Charged Many-Particle Systems, Plenum, New York (1986).

[5] E. Megias, E. Ruiz Arriola and L. L. Salcedo, Phys. Rev. D 75 (2007) 105019 arXiv:hep-ph/0702055.

[6] J. Jankowski, D. Blaschke and H. Grigorian Acta Phys. Polon. Supp. 3 (2010) 747 arXiv:0911.1534 [hep-ph]].

[7] O. Kaczmarek and F. Zantow, arXiv:hep-lat/0506019.

[8] C. Ratti, M. A. Thaler and W. Weise, Phys. Rev. D 73 (2006) 014019 arXiv:hep-ph/0506234.

[9] H. Hansen, W. M. Alberico, A. Beraudo, A. Molinari, M. Nardi and C. Ratti, Phys. Rev. D 75 (2007) 065004 arXiv:hep-ph/0609116.

[10] M. LeBellac, Thermal Field Theory, Cambridge University Press (1996).

[11] A. Beraudo, J. P. Blaizot and C. Ratti, Nucl. Phys. A 806312 (2008).

[12] D. Blaschke et al., Yad. Fiz. 71, 2012 (2008).

[13] P. Petreczky and K. Petrov, Phys. Rev. D 70 (2004) 054503 arXiv:hep-lat/0405009.

[14] O. Kaczmarek, PoS C POD07 (2007) 043 [arXiv:0710.0498 [hep-lat]]. 


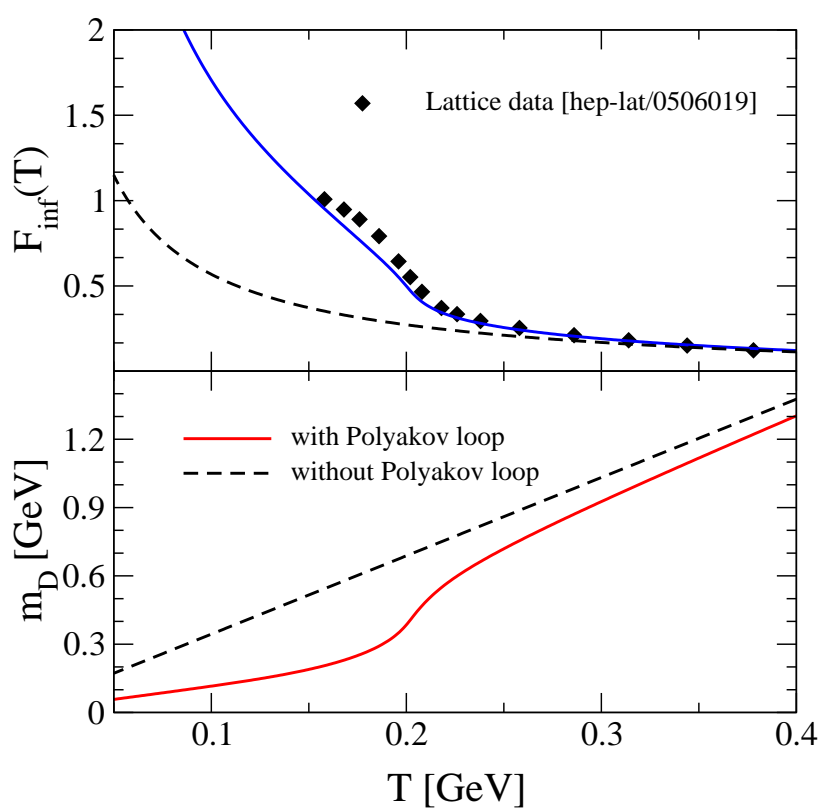

FIG. 1: Lower panel: Temperature dependence of the Debye mass with (solid line) and without (dashed line) inclusion of Polyakov loop. Calculated for $\alpha_{s}=0.471$. Upper panel: Temperature dependence of $F_{1}(r=\infty, T)$ with (solid line) and without (dashed line) inclusion of Polyakov loop, compared to lattice data from Ref. [7]. 